\title{
Limbic links to paranoia: increased resting-state functional connectivity between amygdala, hippocampus and orbitofrontal cortex in schizophrenia patients with paranoia
}

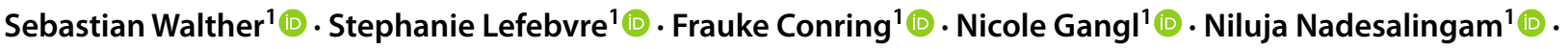 \\ Danai Alexaki ${ }^{1} \cdot$ Florian Wüthrich $^{1}(1) \cdot$ Maximilian Rüter ${ }^{1} \cdot$ Petra V. Viher $^{1} \cdot$ Andrea Federspiel $^{1}{ }^{1} \cdot$ Roland Wiest $^{2}$. \\ Katharina Stegmayer ${ }^{1} \mathbb{D}$
}

Received: 10 May 2021 / Accepted: 22 September 2021 / Published online: 12 October 2021

(c) The Author(s) 2021

\begin{abstract}
Paranoia is a frequent and highly distressing experience in psychosis. Models of paranoia suggest limbic circuit pathology. Here, we tested whether resting-state functional connectivity (rs-fc) in the limbic circuit was altered in schizophrenia patients with current paranoia. We collected MRI scans in 165 subjects including 89 patients with schizophrenia spectrum disorders (schizophrenia, schizoaffective disorder, brief psychotic disorder, schizophreniform disorder) and 76 healthy controls. Paranoia was assessed using a Positive And Negative Syndrome Scale composite score. We tested rs-fc between bilateral nucleus accumbens, hippocampus, amygdala and orbitofrontal cortex between groups and as a function of paranoia severity. Patients with paranoia had increased connectivity between hippocampus and amygdala compared to patients without paranoia. Likewise, paranoia severity was linked to increased connectivity between hippocampus and amygdala. Furthermore, paranoia was associated with increased connectivity between orbitofrontal and medial prefrontal cortex. In addition, patients with paranoia had increased functional connectivity within the frontal hubs of the default mode network compared to healthy controls. These results demonstrate that current paranoia is linked to aberrant connectivity within the core limbic circuit and prefrontal cortex reflecting amplified threat processing and impaired emotion regulation. Future studies will need to explore the association between limbic hyperactivity, paranoid ideation and perceived stress.
\end{abstract}

Keywords Paranoia $\cdot$ Schizophrenia $\cdot$ Resting-state fMRI $\cdot$ Functional connectivity $\cdot$ Limbic system $\cdot$ Amygdala

\section{Introduction}

Most people have a sense of security, i.e. feel generally safe in their social environment [1-3]. The perception of threat, e.g. being harmed or persecuted by others, is extremely distressing. Paranoia describes a spectrum of distressing experiences including mistrust, suspiciousness, ideas of reference, feelings of persecution, and delusions; the extreme end of

Sebastian Walther and Stephanie Lefebvre shared first authorship.

Stephanie Lefebvre

stephanie.lefebvre@upd.unibe.ch

1 Translational Research Center, University Hospital of Psychiatry and Psychotherapy, University of Bern, Bern, Switzerland

2 Institute of Diagnostic and Interventional Neuroradiology, Inselspital, University of Bern, Bern, Switzerland this spectrum includes persecutory delusions that are held with great conviction [3]. While paranoia was conceptualized as a phenomenon of serious psychiatric disorders, research now demonstrates paranoid thinking in the general population with low severity in about one-quarter of the people, but high severity in up to $2 \%$ [4], maintained by a range of cognitive and affective factors (i.e. low self-esteem, worry, sleep dysfunction, perceptual anomalies, and reasoning biases) [3,5]. Delusions with paranoid content are key features of schizophrenia spectrum disorders but also occur in other psychopathologies [6, 7]. Persecutory delusions are found in up to $70 \%$ of schizophrenia patients, cause significant distress, and trigger safety behaviors [8, 9]. Importantly, paranoid delusions have been found to lead to serious acts of violence in first-episode psychosis [9].

While researchers are beginning to unravel the cognitive and emotional factors of delusion formation across different diagnoses, a neurobiological model of paranoia is still 
missing. Paranoia is associated with negative self-beliefs, anxiety, and depression [2] and includes perceptions of immediate threat [3]. Given these negative emotions, the limbic system should play a critical role in the formation and perpetuation of paranoia, particularly the amygdala, hippocampus, and orbitofrontal cortex [10]. Indeed, evidence from structural and perfusion MRI studies in patients with paranoia seems to support this notion. For example, medial temporal lobe structures, particularly the amygdala, show structural alterations in patients with paranoia [11-13]. In addition, two studies reported increased resting-state perfusion of the left amygdala in paranoid patients with schizophrenia, arguing for increased neural activity $[14,15]$. However, it remains unclear whether increased neural activity at rest in the amygdala was related to aberrant functional connectivity in the limbic system, and whether this aberrant connectivity was specific to experiences of paranoia.

In people at clinical high risk for psychosis, persecutory delusions were associated with increased functional connectivity from amygdala to the visual cortex [16]. But evidence on paranoia and functional connectivity in patients with schizophrenia is missing.

Several studies have explored the broader link between resting-state functional connectivity (rs-fc) and delusions generating complex and sometimes conflicting results. In schizophrenia, positive symptom severity, particularly of delusions and hallucinations was associated with rs-fe from thalamus to the middle temporal gyrus [17]. Likewise, effective connectivity at rest between thalamus and the anterior cingulate cortex correlated with positive symptom severity in schizophrenia, particularly the items delusions and persecution [18]. In contrast, functional connectivity between amygdala and precentral gyrus was decreased in first episode patients and linked to increased positive symptom severity, which may include persecutory delusions [19]. At network level, lower connectivity between and within resting-state networks including default mode network (DMN), salience network (SAL), sensorimotor network (SMN), or central executive network (CEN) was associated with severe positive symptoms in schizophrenia [20]. The limited evidence available suggests some association between delusions and alterations (both increases and decreases) in the cerebral resting state. However, the specific neural pattern associated with paranoia remains unknown.

While rs-fc from the amygdala to various brain regions declines with age in typical neurodevelopment, in youth with psychosis this development was deteriorated, leading to increased connectivity between amygdala and striatum, thalamus, ventrolateral prefrontal cortex and occipital cortex [21]. These findings suggest a lack of specialization during neural maturation in subjects with psychosis, which might give rise to increased resting-state activity in the limbic system and paranoid ideation.
In sum, while perfusion MRI studies suggest increased resting-state neural activity in the amygdala in patients with paranoia, we have currently little understanding of the functional connectivity within the limbic structures in patients with current paranoia. Particularly, the connectivity between amygdala, hippocampus, nucleus accumbens or orbitofrontal cortex requires elucidation.

Here, we aimed at exploring associations between aberrant neural activity at rest in the limbic system and current paranoia severity in subjects with schizophrenia. We hypothesized that patients with current paranoia would demonstrate increased functional connectivity at rest between key components of the limbic system when compared to patients without paranoia. In addition, we expect that connectivity within the limbic system was increased as a function of paranoia severity. Therefore, we tested these associations in both categorical and dimensional measures of paranoia.

\section{Subjects and methods}

\section{Subjects}

The current study included 89 patients with schizophrenia spectrum disorders according to DSM-5 criteria (schizophrenia, schizoaffective disorder, brief psychotic disorder, schizophreniform disorder) and 76 healthy controls (Table 1, Part A, Part C) from three different studies that used functional neuroimaging and comprehensive assessment of symptoms in patients with schizophrenia spectrum disorders [OCoPS-P (Overcoming Psychomotor Slowing in Psychosis) ClinicalTrials.gov Identifier: NCT03921450 [22] 10 patients, 17 controls; GNI (neural correlates of gesture deficits in schizophrenia) [23, 24] 46 patients, 44 controls; IPSS (interpersonal space study in schizophrenia) 30 patients, 14 controls]. We recruited patients at the in- and out-patient departments of the University Hospital of Psychiatry, Bern, and healthy controls via advertisement. Healthy controls were matched for age and gender. All participants provided written informed consent. The study protocols adhered to the declaration of Helsinki and were approved by the local ethics committee. General exclusion criteria for all subjects were substance abuse or dependence other than nicotine, history of neurologic disease, head trauma with concurrent loss of consciousness, or history of electroconvulsive treatment, and any MRI counter-indication. Additional exclusion criteria for controls were a history of any psychiatric disorder, as well as any first-degree relatives with schizophrenia or schizoaffective disorder.

We assessed the current psychopathology with the Positive And Negative Syndrome Scale (PANSS) [25]. In addition, we assessed symptom history using the Comprehensive Assessment of Symptoms and History (CASH) 
Table 1 Demographic information

(A): Demographic information of the whole study population

\begin{tabular}{llll}
\hline & Controls & Patients & Comparison \\
\hline$N$ & 76 & 89 & $\mathrm{n} / \mathrm{a}$ \\
Age (years, mean $\pm \mathrm{sd})$ & $36.9 \pm 13.4$ & $37.4 \pm 12.1$ & $t(152.5)=0.24, p=0.81$ \\
Gender & $45 \mathrm{M}$ & $56 \mathrm{M}$ & $\chi^{2}=0.24, p=0.6$ \\
Education (years, mean $\pm \mathrm{sd})$ & $14.9 \pm 3.1$ & $13.2 \pm 2.9$ & $t(151.8)=3.79, p<0.001$ \\
Duration of illness (years, mean $\pm \mathrm{sd})$ & $\mathrm{n} / \mathrm{a}$ & $11.3 \pm 11.2$ & $\mathrm{n} / \mathrm{a}$ \\
Total PANSS (mean $\pm \mathrm{sd})$ & $\mathrm{n} / \mathrm{a}$ & $72.2 \pm 16.3$ & $\mathrm{n} / \mathrm{a}$ \\
Positive PANSS (mean $\pm \mathrm{sd})$ & $\mathrm{n} / \mathrm{a}$ & $17.7 \pm 5.9$ & $\mathrm{n} / \mathrm{a}$ \\
Negative PANSS (mean $\pm \mathrm{sd})$ & $\mathrm{n} / \mathrm{a}$ & $18.3 \pm 6.00$ & $\mathrm{n} / \mathrm{a}$ \\
Medication (OLZ eq.) $(\mathrm{mean} \pm \mathrm{sd})$ & $\mathrm{n} / \mathrm{a}$ & $13.0 \pm 9.7$ & $\mathrm{n} / \mathrm{a}$ \\
\hline
\end{tabular}

(B): Demographic information of the schizophrenia population based on the paranoia score

\begin{tabular}{llll}
\hline & Paranoia & Non-paranoia & Comparison \\
\hline$N$ & 49 & 40 & $t(85.3)=0.65, p=0.52$ \\
Age & $36.7 \pm 12.5$ & $38.3 \pm 11.7$ & $\chi^{2}=0.27, p=0.6$ \\
Gender & $32 \mathrm{M}$ & $24 \mathrm{M}$ & $t(65.38)=0.03, p=0.97$ \\
Duration of illness (years, mean $\pm \mathrm{sd})$ & $11.3 \pm 10.3$ & $11.2 \pm 11.7$ & $t(77.39)=1.14, p=0.26$ \\
Medication & $14.4 \pm 9.3$ & $12.1 \pm 9.9$ & $t(78.5)=12.5, p<0.001$ \\
Paranoia score & $13.2 \pm 3.0$ & $6.9 \pm 1.7$ & $t(77.2)=8.5, p<0.001$ \\
P1 & $4.3 \pm 1.6$ & $2.1 \pm 0.9$ & $t(80.4)=6.0, p<0.001$ \\
P4 & $3.2 \pm 1.0$ & $1.9 \pm 0.9$ & $t(70.7)=6.2, p<0.001$ \\
P5 & $3.0 \pm 1.6$ & $1.4 \pm 0.7$ & $t(80.7)=5.6, p<0.001$ \\
P6 & $2.8 \pm 1.3$ & $1.5 \pm 0.8$ &
\end{tabular}

(C): Comparison across the three groups

\begin{tabular}{llll}
\hline & $D f$ & $F$ value & $\operatorname{Pr}(>F)$ \\
\hline Age & 2 & 0.22 & 0.8027 \\
Years of education & 2 & 7.381 & 0.0008623
\end{tabular}

Post hoc lsmeans (Years of education)

\begin{tabular}{|c|c|c|c|c|c|}
\hline Contrast & Estimate & SE & $d f$ & t.ratio & $p$ value \\
\hline Control一(non-paranoia) & 1.99 & 0.584 & 158 & 3.404 & 0.0024 \\
\hline Control—paranoia & 1.62 & 0.558 & 158 & 2.897 & 0.0119 \\
\hline (Non-paranoia)—paranoia & -0.37 & 0.644 & 158 & -0.574 & 0.8344 \\
\hline
\end{tabular}

$s d$ standard deviation, PANSS Positive And Negative Syndrome Scale, CPZ eq. chlorpromazine-equivalent (mg/day), $M$ male, $\chi^{2}$ chi-square

[26]. All patients were on antipsychotic medication (including thioxanthenes $(n=27)$, olanzapine $(n=21)$, clozapine $(n=11)$, haloperidol $(n=10)$, quietiapine $(n=6)$, amisulpride $(n=5)$, chlorpromazine $(n=2)$, lurasidone $(n=2)$ ). Due to the large variability in the medication type, we calculated the mean olanzapine equivalents (OL eq.) according to Leucht [27] (Table 1, Part A). We performed psychopathology assessments within $48 \mathrm{~h}$ of MRI scanning.

\section{Paranoia assessment}

Multiple studies evaluated paranoia based on different PANSS scores. In the current study, to explore all the possible facets of paranoia, we used a modified version of the composite score established by Williams and collaborators in 2004 [28]. This paranoia score consists of the sum of four items of the PANSS (P1: Delusions, P4: excitement, P5: Grandiosity and P6: Suspiciousness and persecution), 
ranging 4-28. We used this score in two types of analyses: (1) group classification and (2) using the composite paranoia score as a continuous variable.

For the group classification analysis, we compared the rs-fMRI connectivity between "Patients with paranoia" and "Patients without paranoia". To classify the patients, we applied a median split, i.e. a composite paranoia score of 10 (Supplement 1.A, Table 1, Part B). Accordingly, patients with paranoia had a score $\geq 10$, while those without paranoia had a score of $<10$. Subsequently, the two groups were compared on neuroimaging measures. The PANSS score on each of the 4 items was significantly higher in the patients with paranoia compared to the Patients without paranoia (Supplement 1.B). Additionally, we performed correlations between the composite paranoia score and the positive and negative subscore of the PANSS, which are presented in Supplement 1.C. An alternative approach often used in the literature is to aggregate patients according to the PANSS item P6 (suspiciousness) [15]. Accordingly, we repeated the analyses based on the P6 item score, using a cut-off for the group classification (patients with paranoia: P6 score $\geq 3$ ) (see Supplement 3A).

For the continuous variable analysis, we evaluated the rsfMRI connectivity associated with the composite paranoia score. We referred to this analysis as severity analysis.

\section{MRI acquisition and preprocessing}

\section{Acquisition}

Subjects lay horizontally in the MR scanner and their arms rested beside their trunk. To reduce head motion, foam pads were placed around the participants' head and we explicitly instructed participants to avoid head motion.

For 43 patients and 32 healthy controls, the MRI scans were acquired on a 3 T Prisma MRI scanner (Siemens, Germany). The MRI sequences acquired included the following: one T1-weighted MPRAGE scan ( 8 min $22 \mathrm{~s}$ covering 176 sagittal slices, $1 \mathrm{~mm}$ thick, $\mathrm{TR}=5000 \mathrm{~ms}, \mathrm{TE}=2.98 \mathrm{~ms}$, flip angle $1=4^{\circ}$, flip angle $2=5^{\circ}$, voxel size $=1 \times 1 \times 1 \mathrm{~mm}$ ), and one rs-fMRI sequence using a multi-band echoplanar 2D (10 min and $11 \mathrm{~s}$ covering 600 volumes of 72 slices; $2.5 \mathrm{~mm}$ thick, $\mathrm{TR}=1000 \mathrm{~ms}, \mathrm{TE}=37 \mathrm{~ms}$, flip angle $=30^{\circ}$, voxel size $=2.5 \times 2.5 \times 2.5 \mathrm{~mm} \mathrm{FOV}=230 \times 230 \mathrm{~mm}$, GRAPPA $=1$, multiband acceleration factor $=8$ ).

For 46 patients and 44 healthy controls, the MRI scans were acquired on a $3 \mathrm{~T}$ TrioTim MRI scanner (Siemens, Germany). The MRI sequences acquired included the following: T1-weighted MDEFT scan (13 min 43 s covering 176 sagittal slices, $1 \mathrm{~mm}$ thick, $\mathrm{TR}=7.92 \mathrm{~ms}, \mathrm{TE}=2.48 \mathrm{~ms}$, flip angle $=16^{\circ}$, voxel size $1 \times 1 \times 1 \mathrm{~mm}$ ), and one rs-fMRI sequence using a single-band echoplanar $2 \mathrm{D}(8 \mathrm{~min}$ and $40 \mathrm{~s}$ covering 256 volumes of 38 slices; $\mathrm{TR}=2000 \mathrm{~ms}$,
$\mathrm{TE}=30 \mathrm{~ms}$, flip angle $=90^{\circ}$, voxel size $=3.6 \times 3.6 \times 3.0 \mathrm{~mm}$, FOV $=230 \times 230 \mathrm{~mm}$, GRAPPA $=2$ ).

\section{Resting-state fMRI pre-processing}

We analyzed the rs-fMRI data using SPM12 (https://www. fil.ion.ucl.ac.uk/spm/software/spm12) and the CONN toolbox v19 (functional connectivity toolbox; http://www.nitrc. org/projects/conn).

The preprocessing steps included slice-timing correction, motion realignment, co-registration between the functional scans and 3D-T1w scans of each participant and normalization of the functional scans to MNI space using DARTEL and smoothing using a Gaussian filter of $6 \mathrm{~mm}$. In addition, we defined thresholds for participants exclusion due to excessive head motion according to the literature [29-32]: absolute head motion involved translation greater than $2 \mathrm{~mm}$ or rotation greater than $2^{\circ}$ (See Supplement $5 \mathrm{~A}$ ), or if mean frame-wise displacement (FD) (calculated as defined by Power et al. [33]) was greater than $0.5 \mathrm{~mm}$. Using these thresholds resulted in no exclusion of any participants. Furthermore, none of the participants was excluded due to large displacements or spikes in any of the six directions (See Supplement 5). Moreover, one-way ANOVA did not reveal any significant differences in the mean FD between the three groups (patients with paranoia, patients without paranoia and healthy controls, $p=0.352$, See Supplement 5 B). We included the mean FD of each participant as a covariate of non-interest in further analyses. We also performed a denoising step using noise component of white matter and cerebrospinal fluid tissues and motion parameters (12 potential noise components defined from the estimated subject-motion parameters to minimize motion-related BOLD variability, three translation and three rotation parameters plus their associated first-order derivatives) as regressors, a despiking procedure, a linear detrending and band-pass filtering $(0.01-0.1 \mathrm{~Hz})$.

\section{Region of interest definition}

We defined eight regions of interest (ROIs): the bilateral amygdala, hippocampus, nucleus accumbens, and orbitofrontal cortex (OFC). These ROIs were selected from the WFU PickAtlas [34, 35]. The exact coordinates of the center of each ROI and its size were as follows: left amygdala $\left(x=-24, y=-2, z=-19\right.$; size: $\left.1760 \mathrm{~mm}^{3}\right)$, right amygdala $\left(x=27, y=-1, z=-19\right.$; size: $\left.1984 \mathrm{~mm}^{3}\right)$, left hippocampus $\left(x=-30, y=-22, z=-14\right.$; size: $\left.1112 \mathrm{~mm}^{3}\right)$, right hippocampus ( $x=30, y=-22, z=-14$; size: $\left.976 \mathrm{~mm}^{3}\right)$, left nucleus accumbens $\left(x=-14, y=8, z=-12\right.$; size: $\left.984 \mathrm{~mm}^{3}\right)$, right nucleus accumbens $(x=12, y=10, z=-12$; size: 984 $\left.\mathrm{mm}^{3}\right)$, and left OFC $(x=-17, y=46, z=-15$; size: 7704 
$\left.\mathrm{mm}^{3}\right)$, right OFC $\left(x=18, y=47, z=-16\right.$; size: $\left.7976 \mathrm{~mm}^{3}\right)$ (see Supplement 2).

\section{Intracranial volume (ICV) calculation}

Structural brain alterations have been extensively described in schizophrenia [36]. Accordingly, to correct for potential inter-individual head size differences, we used the intracranial volume (ICV) as a covariate of non-interest. The volumes extracted from the segmentation (white matter, grey matter, and cerebrospinal fluid) were summed up to provide an estimate of ICV for each participant.

\section{Resting-state fMRI analysis}

To measure functional changes in rs-fc, we compared the functional connectivity at the region of interest (ROI) level using both ROI-to-ROI and ROI-to-voxels analyses and at the whole-brain level using independent component analysis (ICA, see Supplement 4A).

\section{Region of interest analyses}

We performed two types of ROI analyses: ROI-to-ROI and ROI-to-voxels to compare the three groups: healthy controls, patients with and without paranoia. In the patients' population, we also correlated the rs-fc with the severity of paranoia. In ROI-to-ROI analyses, we aimed to explore rs-fc between the eight ROIs, which are supposed to be strongly interconnected and involved in the neural dysfunction associated with paranoia. We analyzed 28 connections among the 8 ROIs and reported cluster-based inferences [37]. To test the influence of the scanner on the rs-fc, we compared the rs-fc of the healthy controls and of the patients between the two scanner types and added scanner type as covariate of non-interest. To test whether neural activity in these ROIs is linked to neural activity throughout the rest of the brain, we also performed ROI-to-voxels analyses. To compare rs-fc between the three groups: healthy controls, patients with and without paranoia, we performed an ANCOVA including scanner type, age, years of education, mean FD, and ICV as covariates of non-interest. For between patients' comparisons, we used scanner type, mean FD, age, medication dosage and ICV as covariates of non-interest. In addition, to evaluate positive and negative correlations between rs-fc and the severity of paranoia, we used the paranoia score as covariate of interest and scanner type, age, medication dosage and ICV as covariates of non-interest.

In addition, to evaluate any difference in the rs-fc between patients with schizophrenia and healthy controls that might appear outside the ROIs network, we used whole-brain independent component analysis (ICA), the details of this procedure are presented in the supplement (Supplement 4 A).

\section{Statistics}

For demographics analyses, we used $\mathrm{R}$ to perform a Student's $t$ test to compare the age and education between healthy controls and patients with schizophrenia, and a Chisquare repartition test to compare the gender distribution between healthy controls and patients with schizophrenia. Finally, we also performed an ANCOVA to compare age and education between the three groups and a Chi-square repartition test to compare the gender distribution between the three groups. A $p$ value $<0.05$ was considered statistically significant. For ROI-to-ROI analyses, we set the significant threshold as a cluster threshold $\mathrm{p}$ value $\mathrm{qFDR}$ corrected $<0.05$. For ROI-to-voxels analyses, we set a cluster-forming threshold of $p=0.005$ and a $\mathrm{p}$ value $\mathrm{qFDR}$ corrected $<0.05$ for the cluster-wise threshold.

In addition, in the supplement, all of these analyses are presented using the P6 item cut-off instead of the composite paranoia score (see Supplement 3 B).

\section{Results}

No significant differences were observed in the rs-fc between two scanners acquisition neither in healthy subjects nor in patients (Supplementary Fig. 5). [Healthy subjects: no significant difference between the two scanners: $n=76, T(74)$, connection threshold of $p=0.001$ and cluster threshold $p$ value $\mathrm{qFDR}$ corrected $<0.05$. Paranoia patients: no significant difference between the two scanners: $n=57, T(55)$, connection threshold of $p=0.001$ and cluster threshold $p$ value qFDR corrected $<0.05$. Non-paranoia patients: no significant difference between the two scanners: $n=32, T(30)$, connection threshold of $p=0.001$ and cluster threshold $p$ value qFDR corrected $<0.05]$. Accordingly, the data sets from the two scanners were merged and the scanner type was used as a covariate of non-interest in the following analyses.

\section{ROI-to-ROI analysis between the three groups}

The ANCOVA comparing healthy controls, patients with and patients without paranoia demonstrated a significant group effect $\left(F(8,308)=2.55, p_{\mathrm{qFDR}}=0.049\right)$. The post-hoc t-tests revealed an increase of the rs-fc between the hippocampus and the amygdala in the paranoia group compared to the non-paranoia group (Fig. 1, Table 2, Part A). The differences between the control and the two patient groups are not significant. To depict this lack of difference with the healthy controls, we plotted the connectivity values for each ROI-pair with significant group difference across all groups (Fig. 2). Interestingly, healthy controls seem to have an intermediate position in terms of connectivity strength between patients with paranoia and patients without paranoia. 
Fig. 1 ROI-to-ROI rs-fc differences between paranoia and non-paranoia groups. Hippo hippocampus, $R$ right, $L$ left

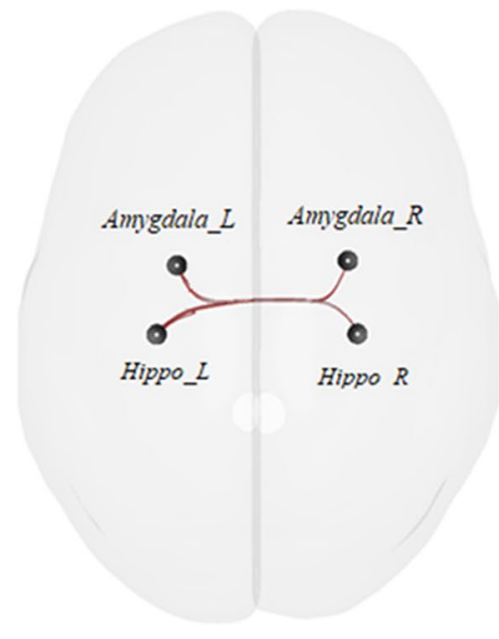

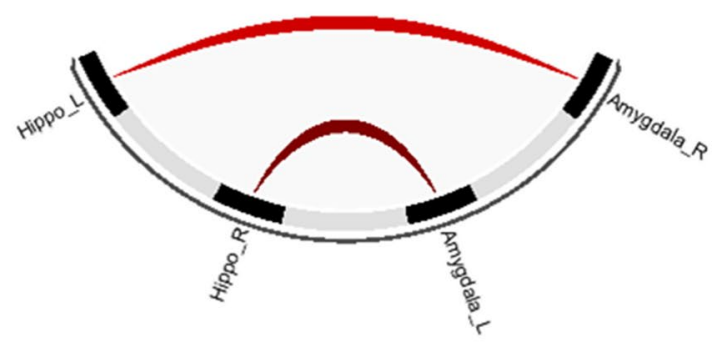

\section{ROI-to-voxels analysis between the three groups}

The ANCOVAs comparing rs-fc between healthy controls, patients with and patients without paranoia demonstrated no significant group effect for any of the seeds.

Due to the intermediate position of the healthy control group in terms of rs-fc (see previous analysis), we performed an additional exploratory analysis comparing only patients with and without paranoia using age, education, scanner type, medication dosage, and ICV as covariates of non-interest. We found an increase of the rs-fc in paranoia patients compared to patients without paranoia between the right OFC and the left BA 39 and between the left OFC and the left BA 47 (Table 2, Part B).

\section{ROI-to-ROI association with paranoia severity}

The ANCOVA exploring the impact of dimensional paranoia ratings on rs-fc on the 28 connections between the 8 ROIs demonstrated significant effects $(F(3,81)=7.10$, $\left.p_{\text {qFDR }}=0.0016\right)$. The post hoc $\mathrm{t}$ tests revealed that an increase in paranoia is associated with increased rs-fc between bilateral hippocampus and bilateral amygdala (Fig. 3, Table 2, Part C).

\section{ROI-to-voxels association with paranoia severity}

The association between paranoia severity and the rs-fc connectivity between every ROI and the rest of the brain was explored. We observed a positive association between the left OFC and the bilateral BA 47 (Table 2, Part D), suggesting that increased paranoia was linked to stronger rs-fc between left OFC and bilateral BA 47.

\section{Additional exploratory analyses}

In the supplementary material, we also describe results for an alternative classification of paranoia using the PANSS P6 item score. When patient groups were categorized according to the P6 item of the PANSS, 12 patients had paranoia and 77 had no paranoia. Due to the imbalance between the two groups, we did not perform the group comparison. Instead, we analyzed the effect of paranoia severity according to P6 on rs-fc, which demonstrated associations between amygdala or hippocampus and temporal cortex (see Supplement 3).

As no significant differences were observed in the rs-fc of the selected ROIs, we performed an additional analysis at the whole-brain level. A whole-brain ICA was used to compare rs-fc between patients with schizophrenia and healthy controls. We identified a reduced functional connectivity in patients compared to controls in the DMN and dorsal attention network (DAN) (Supplement 4B). Likewise, wholebrain ICA of rs-fc indicated multiple DMN alterations in patients with paranoia vs. healthy controls: reduction in the posterior cingulate, but increases in bilateral medial and dorsolateral prefrontal cortex, left angular gyrus, and left middle temporal gyrus (Supplement 4C).

\section{Discussion}

Here, we tested rs-fc within the limbic system in schizophrenia patients with current paranoia. Probing rs-fc between bilateral amygdala, hippocampus, nucleus accumbens, and OFC, we found increased rs-fc between hippocampus and amygdala in patients with paranoia compared to patients without paranoia. This increase in rs-fc between bilateral hippocampus and amygdala was also linked to the severity of paranoia. In additional 
Table 2 Connectivity analyses

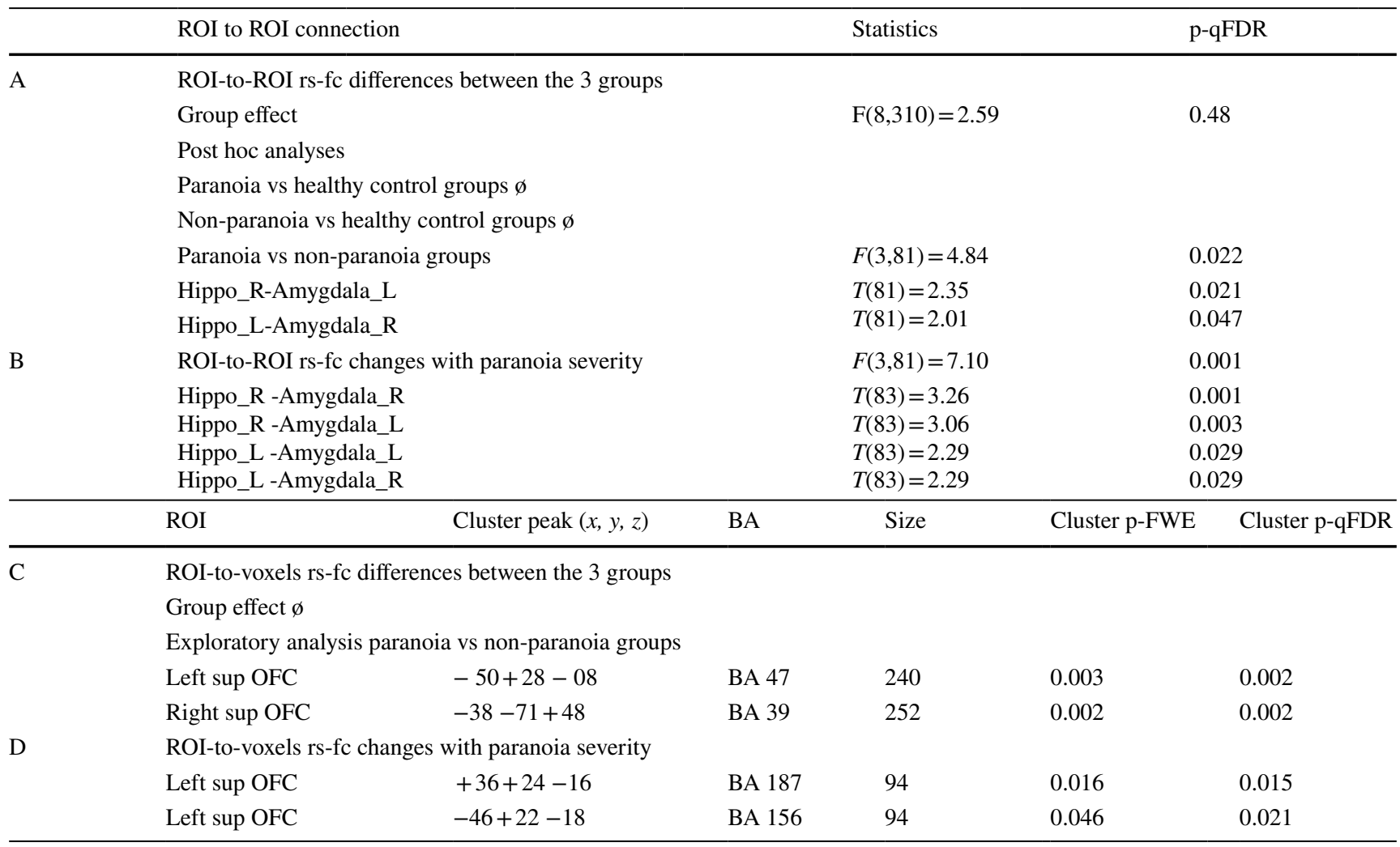

$B A$ broadman area, hippo hippocampus, $O F C$ orbitofrontal cortex, $R$ right, $L$ left

seed-to-voxel analyses, we found the left OFC to be connected to right BA47 in patients with paranoia. Finally, patients with paranoia had increased functional connectivity in prefrontal areas of the DMN when compared to healthy controls. Group differences in rs-fc were corroborated by regression analyses with dimensional assessments of paranoia.

The experience of paranoia is very unpleasant and stressful [3]. Patients engage in multiple safety behaviors when feeling distressed by paranoid ideation [8]. In addition, stress may also trigger further increases in paranoia in both patients with schizophrenia and in subjects vulnerable to psychosis [38-40]. Collectively, these negative emotions are very likely associated with increased neural activity in the limbic system. Indeed, two perfusion MRI studies demonstrated increased resting-state activity of the amygdala in paranoid patients with schizophrenia [14, 15]. Increased resting-state perfusion indicates stronger metabolic activity. In the resting-state, cerebral perfusion and functional connectivity are highly correlated particularly in the DMN and prefrontal cortex, while this correlation strength increases with task demands [41]. Thus, we could expect increased rs-fc from the amygdala in patients with current paranoia.
Threat processing engages ventral hippocampus, amygdala, and orbitofrontal cortex in fMRI tasks in healthy subjects [42-44]. Exposure to threat activated the amygdala for example during a computer gaming predator-prey task [40], while watching short video clips of threatening action [41], and engaged anterior hippocampus during an approachavoidance conflict under varying levels of potential threat [39]. Likewise, testing patients with selective hippocampal lesions show altered avoidance behavior and threat processing [39]. In addition, the nucleus accumbens is important for processing avoidance [45]. Finally, the ventromedial and dorsolateral prefrontal cortices are critical in regulating the activity of this limbic network [46, 47].

Increased rs-fc between components of the subcortical amygdala pathway (hippocampus-amygdala) in paranoia suggests that this pathway is particularly amplified in paranoia, which might be critical to experience stress upon threat. This over-excitation could result from chronic neural alterations in the pathway, as suggested by converging animal models and neuroimaging studies. Indeed, rodent models of schizophrenia consider the ventral hippocampus to be particularly vulnerable to threat induced stress, which is probably due to the distinct maturational profile 

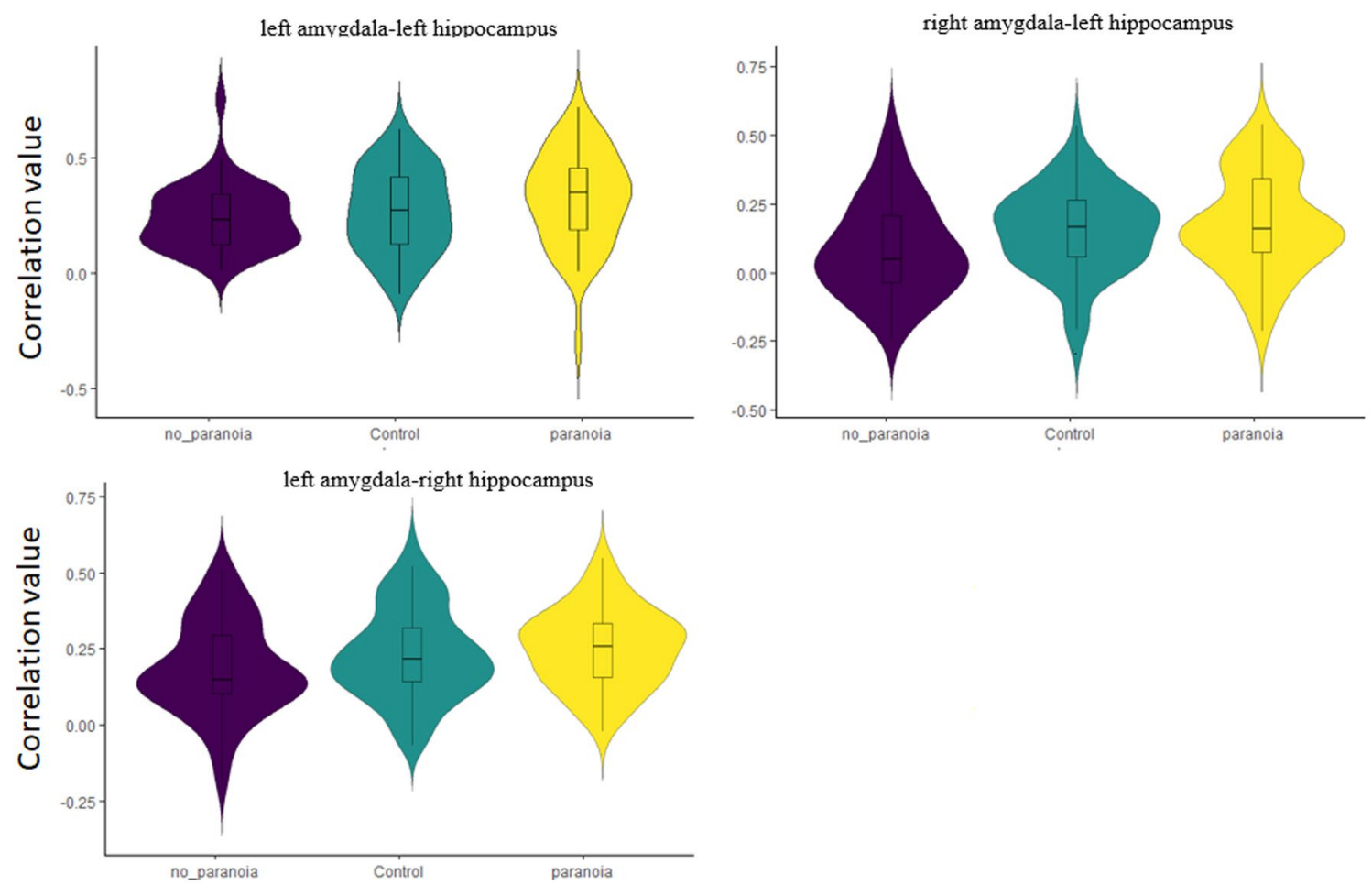

Fig. 2 Violin distributions of the ROI-to-ROI correlation value for each subgroup in the pairs of interest. In each violin, a box plot is added. The center line represents the median value, the lower bound

of the box represents the 25th percentile, the upper bound of the box the 75th percentile, and the whiskers represent three times the interquartile range

Fig. 3 ROI-to-ROI rs-fc changes with paranoia severity maps. Hippo hippocampus, $R$ right, $L$ left
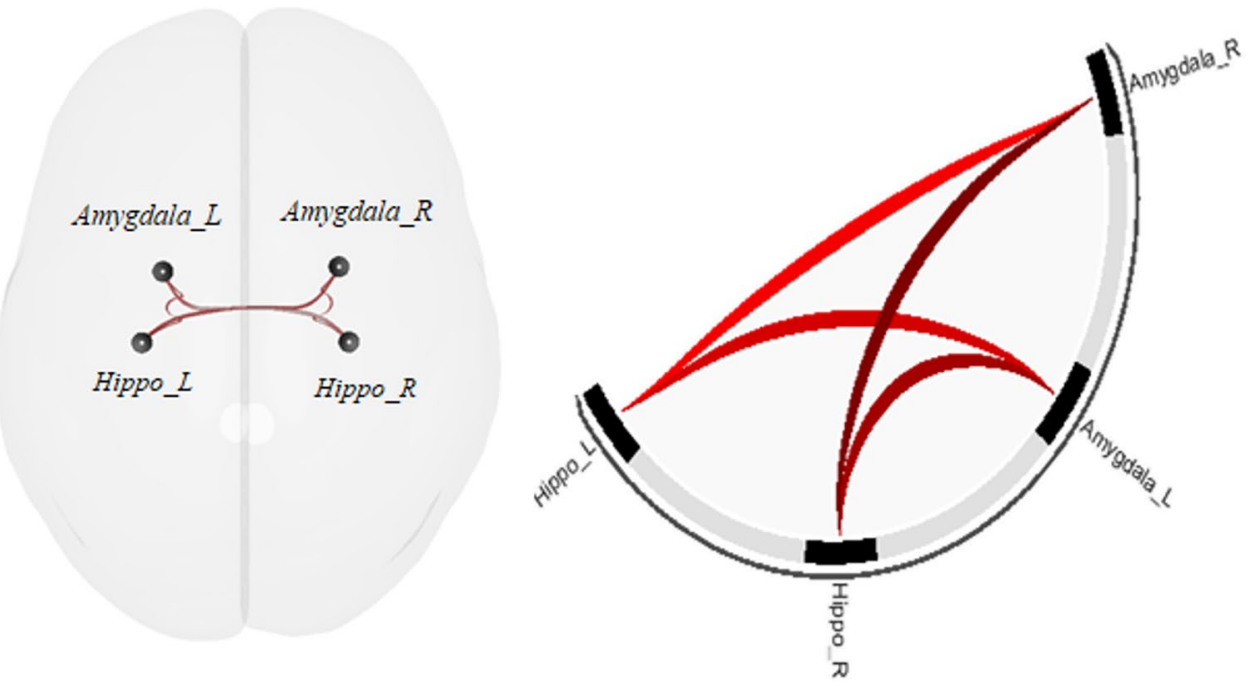

of fast-spiking parvalbumin interneurons (PIV) [48-50]. If threat-induced stress was sufficiently strong, it may trigger hippocampal PIV pathology including cell death [48]. To have this impact, threat either has to be extremely severe or needs to be experienced as extraordinary severe (e.g. in paranoia). Various stressors may activate glutamatergic projections from the amygdala to the hippocampal PIV in schizophrenia; strong stressors interact directly while mild stressors may exert the effect when amygdala regulation from prefrontal cortex is compromised [48]. Along this line, dysfunctional parvalbumin containing inhibitory interneurons seems to drive hippocampal hypermetabolism in schizophrenia [51]. Indeed, increased perfusion as an indicator of hypermetabolism as well as concurrent atrophy 
have been reported in the hippocampus in early psychosis [52-55] and subjects at risk [56], in which social stress may particularly attenuate positive symptoms [57]. Collectively, our functional connectivity findings are in line with studies demonstrating increased neural activity in the amygdala in patients experiencing paranoia $[14,15]$. In addition, findings extend previous knowledge in demonstrating increased connectivity in key components of the limbic system compared to patients without paranoia. Furthermore, we may speculate that the experience of paranoia might also be linked to inefficient stress regulation. However, this proposed link between stress, limbic system hyperactivity, and paranoia requires further work to elucidate the association.

Outside the core limbic circuit, paranoia was also associated with increased rs-fc within the orbital and medial prefrontal cortex (OMPFC including the OFC and BA 47) and between the OFC and the parietal cortex (BA 39, angular gyrus). In general, the OMPFC constitutes a substantial portion of the cerebral cortex in primates. Although its function is not yet fully understood, studies in both non-human primates and humans suggest a role in decision-making and guidance of emotional and reward-related behaviours [58]. Furthermore, BA47 is particularly involved in reappraising negative emotions and suppressing subsequent behaviors [59]. Thus, altered connectivity of the OMPFC in paranoia may either reflect cognitive threat processing and delusion formation, as OMPFC interacts with ventral hippocampus, basal amygdala, and medial prefrontal cortex. A task-based fMRI study argues for a role in cognitive threat processing [60]. When healthy subjects found threatening verbal statements to relate to themselves BA47 was active, while attention to threatening statements was linked to BA39 activity. Thus, both brain regions could be relevant for the formation of paranoid delusions. In line with these findings, paranoia severity was associated with rs-fc increases between OFC and BA47 in our study. However, as we investigated the cerebral resting state, we can only speculate whether increased connectivity suggests aberrant stress regulation, delusion formation or both. Therapeutic efforts may aim at reducing neural hyperconnectivity and hyperactivity in the limbic system.

The experience of paranoia may trigger safety behaviors, such as avoidance, which has been linked to Ncl. accumbens function $[8,45,61]$. We reported previously that structural connectivity between amygdala and $\mathrm{Ncl}$. accumbens was associated with the severity of positive symptoms in schizophrenia [11]. Likewise, grandiosity and paranoid threat were linked to structural alterations in the pathway from the ventral tegmentum that includes Ncl. accumbens [12]. However, the Ncl. accumbens was not related to the presence or severity of paranoia using rs-fc. We may speculate that rs-fc may better reflect the experience of stress and threat in paranoia than paranoia-related avoidance.
Aberrant rs- $\mathrm{fc}$ in the DMN has become a frequent finding in schizophrenia. However, the direction of change and the DMN associations with schizophrenia symptoms remain subject of debate [62,63]. DMN activity has been explained with self-referential processing, emotion regulation, and recollection of prior experiences [64]. We may speculate that self-referential processes and emotion regulation within the frontal DMN hubs are both critically involved in sustaining delusional threat beliefs that feed paranoia. Our findings in patients with current paranoia are in line with previous reports, demonstrating a correlation of frontal DMN connectivity at rest with positive symptoms, i.e. delusions and hallucinations [63, $65,66]$. We detected increased $r s-f c$ in prefrontal cortex components of the DMN in paranoia patients compared to healthy controls. Likewise, our finding of reduced DMN connectivity at rest in schizophrenia compared to controls is in line with a meta-analysis and parts of the literature [65-67]. While altered connectivity within the DMN may not explain symptoms conclusively, it's interaction with other cerebral networks is suggested to contribute to the clinical phenomena in schizophrenia [62, 68-70].

In a broader context, specific behaviors in schizophrenia are linked to patterns of brain alterations including grey matter reductions, increased resting-state neural activity as well as increased structural and functional connectivity within the key networks. In fact, in schizophrenia patients with psychomotor inhibition, we found reduced grey matter volume and increased resting-state perfusion in the supplementary motor area across samples, who in turn demonstrated increased rs-fc and structural connectivity with other motor areas of the brain [71-75]. The brain alterations in patients with paranoia follow a similar pattern: reduced grey matter volumes in amygdala and ventral striatum $[13,76]$, increased restingstate perfusion of the amygdala [14, 15], and increased rs-fc within a limbic network. Thus, specific alterations of cerebral circuit physiology may give rise to distinct symptoms in psychosis.

The most pronounced differences were noted between patients with and without paranoia. In the ROI-to-ROI or ROI-to-voxel analyses we failed to find differences between patients and controls, while we found differences at the network level (e.g. DMN). This pattern of findings argues for enormous biological variance between patients with schizophrenia that is even exceeding the differences between patients and controls, which is in line with larger analyses of structural brain changes in schizophrenia [77]. Therefore, our findings support a dimensional view on schizophrenia pathology that takes symptom dimensions into account. In the present study, we explored paranoia severity using a composite score based on 4 selected PANSS items (delusions, excitement, grandiosity and 
suspiciousness and persecution) as in previous studies [28]. As depicted in Supplentary 1C, the composite paranoia score correlates with the PANSS positive subscore but does not correlate with the PANSS negative score, which is in line with the idea that paranoia contributes to positive psychotic symptoms $[78,79]$.

\section{Limitations}

The findings of the current study should be interpreted in light of some limitations. First, data of three studies in psychosis were pooled for this analysis. These data were acquired with two different $3 \mathrm{~T}$ Siemens scanners at Bern University. The differences in the acquisition parameters between the two scanners are mostly observed on the rsfMRI sequences. The two sequences presented a different type of acquisition (single vs multiband), TR, volume numbers and voxel sizes. Even if some of these differences are corrected after preprocessing and first-level analysis, one could argue that the difference in acquisition might lead to a difference in the observed functional connectivity. However, in this study, the rs-fMRI analysis accounted for the acquisition differences between scanners, and the results remained unchanged after correction. Moreover, the group comparisons between the two scanners demonstrated no significant difference. One additional way to unambiguously check the inter-scanner reliability would be to perform the analyses separately for each acquisition type. However, due to the strong variability in the paranoiac symptoms and severity, large sample sizes are needed to catch the whole picture. In future studies, a more homogenous design and imaging acquisition parameters would be needed to confirm the present results. Second, despite the similarities between the three studies, specific rating scales for the assessment of paranoia have not been consistently applied. Specific scales on paranoia, either expert-rated or self-report are likely to be more sensitive in assessing paranoia severity than the PANSS score [28]. Third, patients were on antipsychotic medication, potentially influencing both paranoia severity and rs-fc. Moreover, the patients were all on different combinations of antipsychotic medication. Even if we used an equivalence scale (olanzapine equivalent) to evaluate the medication dosage of each patient and controlled for it in our statistical models, it is worth noting that equivalence scales can not depict the entire medication profile of the patients. With new medication coming, several antipsychotic drugs are missing from the equivalence scales and the establishment of these scales also suffers from some limitations such as the lack of very large randomized controlled trials to define dose equivalencies. Fourth, we focused on subjects with clear paranoia, neglecting the transdiagnostic similarities or subclinical paranoid experiences in the general population. Future studies should therefore test the associations of functional connectivity with dimensional measures of paranoia to capitalize on the whole spectrum of the paranoia continuum. Fifth, other studies on paranoia have included subjects with stronger symptom severity $[13,15]$. But our sample was heterogeneous in terms of paranoia severity, thus particularly suited for dimensional assessments. In fact, the results of the regression analyses closely aligned with those of the group comparisons. Sixth, in the present study we focused exclusively on functional connectivity, as more homogenous population and design would be needed to test effective connectivity in the limbic system in paranoia. However, the present results provide a solid background for future studies. Seventh, our study included 89 patients with schizophrenia spectrum disorders (schizophrenia, schizoaffective disorder, brief psychotic disorder, schizophreniform disorder), but was not designed to analyse these groups of patients separately. Thus our results do not necessarily generalize to all schizophrenia spectrum disorders. Finally, we had no physiological data documenting stress in subjects with paranoia. Future investigations will allow integrating physiology with self-reported paranoia and brain imaging.

\section{Conclusion}

The current study investigated rs-fc in patients with paranoia. Increased connectivity between hippocampus and amygdala as well as alterations in the DMN in patients with paranoia argue for dysfunctional threat processing in these patients. Instead of indicating a general phenomenon, these alterations in functional connectivity are specific to schizophrenia patients experiencing paranoia.

Supplementary Information The online version contains supplementary material available at https://doi.org/10.1007/s00406-021-01337-w.

Acknowledgements This work was supported by the Swiss National Science Foundation (grants \#152619 and \#182469 to SW, PZPGP3_180022 to KS), the Bangerter Rhyner Foundation (SW), and the Adrian and Simone Frutiger Foundation (KS).

Funding Open Access funding provided by Universität Bern.

Data availability The statistical maps that support the findings of this study are available from the corresponding author upon reasonable request.

\section{Declarations}

Conflict of interest SW has received honoraria from Neurolite, Janssen, Lundbeck, Otsuka, and Sunovion. KS has received honoraria from Lundbeck and Sunovion. SL, FC, NG, NN, DA, FW, MR, PVV, AF and RW reported no biomedical financial interests or potential conflicts of interest. 
Open Access This article is licensed under a Creative Commons Attribution 4.0 International License, which permits use, sharing, adaptation, distribution and reproduction in any medium or format, as long as you give appropriate credit to the original author(s) and the source, provide a link to the Creative Commons licence, and indicate if changes were made. The images or other third party material in this article are included in the article's Creative Commons licence, unless indicated otherwise in a credit line to the material. If material is not included in the article's Creative Commons licence and your intended use is not permitted by statutory regulation or exceeds the permitted use, you will need to obtain permission directly from the copyright holder. To view a copy of this licence, visit http://creativecommons.org/licenses/by/4.0/.

\section{References}

1. Mikulincer M, Shaver PR (2012) An attachment perspective on psychopathology. World Psychiatry 11(1):11-15

2. Bird JC et al (2019) Adolescent paranoia: prevalence, structure, and causal mechanisms. Schizophr Bull 45(5):1134-1142

3. Freeman D (2016) Persecutory delusions: a cognitive perspective on understanding and treatment. Lancet Psychiatry 3(7):685-692

4. Bebbington PE et al (2013) The structure of paranoia in the general population. Br J Psychiatry 202:419-427

5. Freeman D et al (2019) Automated psychological therapy using virtual reality (VR) for patients with persecutory delusions: study protocol for a single-blind parallel-group randomised controlled trial (THRIVE). Trials 20(1):87

6. Bebbington P, Freeman D (2017) Transdiagnostic extension of delusions: schizophrenia and beyond. Schizophr Bull 43(2):273-282

7. Bentall RP et al (2009) The cognitive and affective structure of paranoid delusions: a transdiagnostic investigation of patients with schizophrenia spectrum disorders and depression. Arch Gen Psychiatry 66(3):236-247

8. Freeman D et al (2007) Acting on persecutory delusions: the importance of safety seeking. Behav Res Ther 45(1):89-99

9. Coid JW et al (2013) The relationship between delusions and violence: findings from the East London first episode psychosis study. JAMA Psychiatr 70(5):465-471

10. Strik W et al (2017) Systems neuroscience of psychosis: mapping schizophrenia symptoms onto brain systems. Neuropsychobiology 75(3):100-116

11. Bracht $\mathrm{T}$ et al (2014) White matter pathway organization of the reward system is related to positive and negative symptoms in schizophrenia. Schizophr Res 153(1-3):136-142

12. Bracht $T$ et al (2019) Increased structural connectivity of the medial forebrain bundle in schizophrenia spectrum disorders is associated with delusions of paranoid threat and grandiosity. Neuroimage Clin 24:102044

13. Stegmayer K et al (2014) Ventral striatum gray matter density reduction in patients with schizophrenia and psychotic emotional dysregulation. Neuroimage Clin 4:232-239

14. Stegmayer K et al (2017) Specific cerebral perfusion patterns in three schizophrenia symptom dimensions. Schizophr Res (2017)

15. Pinkham AE et al (2015) Amygdala hyperactivity at rest in paranoid individuals with schizophrenia. Am J Psychiatry 172(8):784-792

16. DeCross SN et al (2020) Increased amygdala-visual cortex connectivity in youth with persecutory ideation. Psychol Med 50(2):273-283

17. Ferri $\mathbf{J}$ et al (2018) Resting-state thalamic dysconnectivity in schizophrenia and relationships with symptoms. Psychol Med 48(15):2492-2499
18. Csukly G et al (2020) Fronto-thalamic structural and effective connectivity and delusions in schizophrenia: a combined DTI/ DCM study. Psychol Med 2020:1-11

19. Zhang M et al (2020) Abnormal amygdala subregional-sensorimotor connectivity correlates with positive symptom in schizophrenia. Neuroimage Clin 26:102218

20. Lee WH et al (2018) Resting-state network connectivity and metastability predict clinical symptoms in schizophrenia. Schizophr Res 201:208-216

21. Jalbrzikowski M et al (2019) Age-associated deviations of amygdala functional connectivity in youths with psychosis spectrum disorders: relevance to psychotic symptoms. Am J Psychiatry 176(3):196-207

22. Walther $\mathrm{S}$ et al (2020) Inhibitory repetitive transcranial magnetic stimulation to treat psychomotor slowing: a transdiagnostic, mechanism-based randomized double-blind controlled trial. Schizophr Bull Open 1(1):1

23. Wuthrich F et al (2020) Dysbalanced resting-state functional connectivity within the praxis network is linked to gesture deficits in schizophrenia. Schizophr Bull 46(4):905-915

24. Walther $S$ et al (2015) Nonverbal social communication and gesture control in schizophrenia. Schizophr Bull 41(2):338-345

25. Kay SR, Fiszbein A, Opler LA (1987) The positive and negative syndrome scale (PANSS) for schizophrenia. Schizophr Bull 13(2):261-276

26. Andreasen NC, Flaum M, Arndt S (1992) The comprehensive assessment of symptoms and history (CASH): an instrument for assessing diagnosis and psychopathology. Arch Gen Psychiatry 49(8):615-623

27. Leucht $S$ et al (2015) Dose equivalents for second-generation antipsychotic drugs: the classical mean dose method. Schizophr Bull 41(6):1397-1402

28. Williams LM et al (2004) Dysregulation of arousal and amygdala-prefrontal systems in paranoid schizophrenia. Am J Psychiatry 161(3):480-489

29. Li P et al (2020) Association between functional and structural connectivity of the corticostriatal network in people with schizophrenia and unaffected first-degree relatives. J Psychiatry Neurosci 45(6):395-405

30. Stripeikyte G. et al (2021) Fronto-temporal disconnection within the presence hallucination network in psychotic patients with passivity experiences. Schizophr Bull (2021)

31. Faria AV et al (2021) Multimodal MRI assessment for first episode psychosis: a major change in the thalamus and an efficient stratification of a subgroup. Hum Brain Mapp 42(4):1034-1053

32. Gangadin SS et al (2021) Reduced resting state functional connectivity in the hippocampus-midbrain-striatum network of schizophrenia patients. J Psychiatr Res 138:83-88

33. Power JD et al (2012) Spurious but systematic correlations in functional connectivity MRI networks arise from subject motion. Neuroimage 59(3):2142-2154

34. Maldjian JA, Laurienti PJ, Burdette JH (2004) Precentral gyrus discrepancy in electronic versions of the Talairach atlas. Neuroimage 21(1):450-455

35. Maldjian JA et al (2003) An automated method for neuroanatomic and cytoarchitectonic atlas-based interrogation of fMRI data sets. Neuroimage 19(3):1233-1239

36. Haijma SV et al (2013) Brain volumes in schizophrenia: a meta-analysis in over 18000 subjects. Schizophr Bull 39(5):1129-1138

37. Jafri MJ et al (2008) A method for functional network connectivity among spatially independent resting-state components in schizophrenia. Neuroimage 39(4):1666-1681

38. Lincoln TM et al (2010) The effect of state anxiety on paranoid ideation and jumping to conclusions. An experimental investigation. Schizophr Bull 36(6):1140-1148 
39. Moritz S et al (2011) Elucidating the black box from stress to paranoia. Schizophr Bull 37(6):1311-1317

40. Freeman D et al (2015) The stress of the street for patients with persecutory delusions: a test of the symptomatic and psychological effects of going outside into a busy urban area. Schizophr Bull 41(4):971-979

41. Liang $X$ et al (2013) Coupling of functional connectivity and regional cerebral blood flow reveals a physiological basis for network hubs of the human brain. Proc Natl Acad Sci U S A 110(5):1929-1934

42. Bach DR et al (2014) Human hippocampus arbitrates approachavoidance conflict. Curr Biol 24(5):541-547

43. Gold AL, Morey RA, McCarthy G (2015) Amygdala-prefrontal cortex functional connectivity during threat-induced anxiety and goal distraction. Biol Psychiatry 77(4):394-403

44. Pichon S, de Gelder B, Grezes J (2012) Threat prompts defensive brain responses independently of attentional control. Cereb Cortex 22(2):274-285

45. Ramirez F et al (2015) Active avoidance requires a serial basal amygdala to nucleus accumbens shell circuit. J Neurosci 35(8):3470-3477

46. LeDoux JE, Pine DS (2016) Using neuroscience to help understand fear and anxiety: a two-system framework. Am J Psychiatry 173(11):1083-1093

47. Schiller D, Delgado MR (2010) Overlapping neural systems mediating extinction, reversal and regulation of fear. Trends Cogn Sci 14(6):268-276

48. Gomes FV, Zhu X, Grace AA (2019) Stress during critical periods of development and risk for schizophrenia. Schizophr Res 213:107-113

49. Perkins DO, Jeffries CD, Do KQ (2020) Potential roles of redox dysregulation in the development of schizophrenia. Biol Psychiatry 88(4):326-336

50. Lieberman JA et al (2018) Hippocampal dysfunction in the pathophysiology of schizophrenia: a selective review and hypothesis for early detection and intervention. Mol Psychiatry 23(8):1764-1772

51. Sonnenschein SF, Gomes FV, Grace AA (2020) Dysregulation of midbrain dopamine system and the pathophysiology of schizophrenia. Front Psychiatry 11:613

52. Schobel SA et al (2013) Imaging patients with psychosis and a mouse model establishes a spreading pattern of hippocampal dysfunction and implicates glutamate as a driver. Neuron 78(1):81-93

53. Modinos G et al (2018) Prefrontal GABA levels, hippocampal resting perfusion and the risk of psychosis. Neuropsychopharmacology 43(13):2652-2659

54. Allen P et al (2016) Resting hyperperfusion of the hippocampus, midbrain, and basal ganglia in people at high risk for psychosis. Am J Psychiatry 173(4):392-399

55. McHugo M et al (2019) Hyperactivity and reduced activation of anterior hippocampus in early psychosis. Am J Psychiatry 176(12):1030-1038

56. Wolthusen RPF et al (2018) Correlation between levels of delusional beliefs and perfusion of the hippocampus and an associated network in a non-help-seeking population. Biol Psychiatry Cogn Neurosci Neuroimaging 3(2):178-186

57. Millman ZB et al (2018) Perceived social stress and symptom severity among help-seeking adolescents with versus without clinical high-risk for psychosis. Schizophr Res 192:364-370

58. Ongur D, Ferry AT, Price JL (2003) Architectonic subdivision of the human orbital and medial prefrontal cortex. J Comp Neurol 460(3):425-449
59. Quirk GJ, Beer JS (2006) Prefrontal involvement in the regulation of emotion: convergence of rat and human studies. Curr Opin Neurobiol 16(6):723-727

60. Blackwood NJ et al (2000) Imaging attentional and attributional bias: an fMRI approach to the paranoid delusion. Psychol Med 30(4):873-883

61. Schlund MW et al (2013) Neuroimaging the temporal dynamics of human avoidance to sustained threat. Behav Brain Res 257:148-155

62. Jardri R et al (2013) The neurodynamic organization of modalitydependent hallucinations. Cereb Cortex 23(5):1108-1117

63. Whitfield-Gabrieli S et al (2009) Hyperactivity and hyperconnectivity of the default network in schizophrenia and in first-degree relatives of persons with schizophrenia. Proc Natl Acad Sci U S A 106(4):1279-1284

64. Raichle ME (2015) The brain's default mode network. Annu Rev Neurosci 38:433-447

65. Camchong $\mathbf{J}$ et al (2011) Altered functional and anatomical connectivity in schizophrenia. Schizophr Bull 37(3):640-650

66. Rotarska-Jagiela A et al (2010) Resting-state functional network correlates of psychotic symptoms in schizophrenia. Schizophr Res 117(1):21-30

67. Doucet GE et al (2020) Transdiagnostic and disease-specific abnormalities in the default-mode network hubs in psychiatric disorders: a meta-analysis of resting-state functional imaging studies. Eur Psychiatry 63(1):e57

68. Lefort-Besnard J et al (2018) Different shades of default mode disturbance in schizophrenia: subnodal covariance estimation in structure and function. Hum Brain Mapp 39(2):644-661

69. Kindler J et al (2015) Static and dynamic characteristics of cerebral blood flow during the resting state in schizophrenia. Schizophr Bull 41(1):163-170

70. Lefebvre $S$ et al (2016) Network dynamics during the different stages of hallucinations in schizophrenia. Hum Brain Mapp 37(7):2571-2586

71. Bracht $\mathrm{T}$ et al (2013) Altered cortico-basal ganglia motor pathways reflect reduced volitional motor activity in schizophrenia. Schizophr Res 143(2-3):269-276

72. Walther $\mathrm{S}$ et al (2017) Resting-state hyperperfusion of the supplementary motor area in catatonia. Schizophr Bull 43(5):972-981

73. Walther $\mathrm{S}$ et al (2017) Aberrant hyperconnectivity in the motor system at rest is linked to motor abnormalities in schizophrenia spectrum disorders. Schizophr Bull 43(5):982-992

74. Stegmayer K et al (2014) Supplementary motor area (SMA) volume is associated with psychotic aberrant motor behaviour of patients with schizophrenia. Psychiatry Res 223(1):49-51

75. Viher PV et al (2020) Altered diffusion in motor white matter tracts in psychosis patients with catatonia. Schizophr Res 220:210-217

76. Wolf RC et al (2021) Aberrant gray matter volume and cortical surface in paranoid-type delusional disorder. Neuropsychobiology 2020:1-10

77. Alnaes D et al (2019) Brain heterogeneity in schizophrenia and its association with polygenic risk. JAMA Psychiat 76(7):739-748

78. Pankow A et al (2012) Neurobiological correlates of delusion: beyond the salience attribution hypothesis. Neuropsychobiology 66(1):33-43

79. Abi-Dargham A et al (2000) Increased baseline occupancy of D2 receptors by dopamine in schizophrenia. Proc Natl Acad Sci U S A 97(14):8104-8109 